\title{
ON SHAKEDOWN OF ELASTOPLASTIC SHELLS
}

\author{
By \\ HELMUT STUMPF AND LE KHANH CHAU \\ Ruhr-Universität Bochum, Bochum, Germany
}

\begin{abstract}
An asymptotic theory of adaptation for elastoplastic shells under a variable loading is proposed. The hypothesis of membrane state of an elastic response is used to reduce the three-dimensional variational problems for shakedown factor to two-dimensional ones. The duality and the possibility of algebraization allow the membrane shell shakedown theory to be analytically solvable in many interesting cases. The asymptotic accuracy of the constructed membrane approximation is proved.
\end{abstract}

1. Introduction. Let us consider a domain $\mathscr{B}$ of the three-dimensional Euclidean space $\mathscr{E}^{3}$, whose points are described by

$$
\mathbf{X}\left(\xi^{\alpha}, \xi\right)=\mathbf{r}\left(\xi^{\alpha}\right)+\xi \mathbf{n}\left(\xi^{\alpha}\right), \quad|\xi| \leq h / 2 .
$$

Here $\mathbf{r}\left(\xi^{\alpha}\right)$ denotes the position vector of points of a smooth surface $\Omega$ bounded by the smooth contour $\Gamma$, and $\mathbf{n}\left(\xi^{\alpha}\right)$ is the unit vector normal to $\Omega$. An elastoplastic body occupying the domain $\mathscr{B}$ in its undeformed configuration is called an elastoplastic shell with middle surface $\Omega$ and thickness $h$.

Let the elastoplastic shell be subjected to a cyclic loading, specified either by body loads and surface tractions or, equivalently, by the stress field $\stackrel{\mathrm{e}}{\boldsymbol{\sigma}}(t)$ with a time period $T$. This symmetric tensor field describes the purely elastic response of an elastic comparison shell of the same shape with the same compliance matrix to the given loading history. The static shakedown factor is then defined as a solution of an optimization problem (Melan [1])

$$
\begin{array}{r}
\alpha=\sup \{v \mid v \in \mathbb{R}, \quad \exists \boldsymbol{\rho} \in \mathcal{J}, \forall t \in[0, T] \\
v(\boldsymbol{\sigma}+\boldsymbol{\rho}) \in \mathscr{C}\} .
\end{array}
$$

Here $\mathscr{J}$ corresponds to the space of all time-independent self-equilibrated residual stress fields $\rho$ such that

$$
\text { Div } \boldsymbol{\rho}=0, \quad \boldsymbol{\rho}^{\mathrm{T}}=\boldsymbol{\rho}, \quad \boldsymbol{\rho} \mathbf{n}=0 \quad \text { on } \partial \mathscr{B}_{T},
$$

with $\partial \mathscr{B}_{T}$ the part of the boundary $\partial \mathscr{B}$ where the surface tractions are prescribed. The notation used in the text is standard with Div the divergence operator and $\mathbf{n}$ eceived July 6, 1990. 
the unit vector normal to $\partial \mathscr{B}$. The convex set $\mathscr{C} \subset \mathbb{R}^{6}$ (with $0 \in \mathscr{C}$ ) consists of all statically admissible stress tensors, which do not violate the yield condition.

The dual (with respect to (2)) variational problem leads to the kinematical shakedown factor (Koiter [2], Gokhfeld [3], Sawczuk [4])

$$
\beta=\inf \left\{\int_{0}^{T} \int_{\mathscr{B}} \varphi^{*}\left(\mathbf{e}^{p}\right) d X d t \mid \int_{0}^{T} \mathbf{e}^{p} d t \in \mathscr{K}, \int_{0}^{T} \int_{\mathscr{B}} \underset{\boldsymbol{e}}{\boldsymbol{e}} \cdot \mathbf{e}^{p} d X d t=1\right\} .
$$

Here the symmetric tensor $\mathbf{e}^{p}=\dot{\boldsymbol{\epsilon}}^{p}$ describes the plastic strain rate. The dual (with respect to $\mathscr{J}$ ) space $\mathscr{K}$ consists of all kinematically admissible strain fields $\epsilon^{p}$ such that

$$
\boldsymbol{\epsilon}^{p}=\frac{1}{2}\left[\nabla \mathbf{w}+(\nabla \mathbf{w})^{\mathrm{T}}\right], \quad \mathbf{w}=0 \quad \text { on } \partial \mathscr{B}_{x},
$$

with $\partial \mathscr{B}_{x}$ the part of $\partial \mathscr{B}$ where displacements are prescribed, $\partial \mathscr{B}_{x} \cap \partial \mathscr{B}_{T}=\varnothing$, $\overline{\partial \mathscr{B}_{x} \cup \partial \mathscr{B}_{T}}=\partial \mathscr{B}$. The function $\varphi^{*}\left(\mathbf{e}^{p}\right)$ in (4) is defined by the Fenchel transformation of the indicator-function $\varphi_{\mathscr{E}}(\sigma)$ of the convex set $\mathscr{C}$ :

$$
\varphi^{*}\left(\mathbf{e}^{p}\right)=\sup _{\boldsymbol{\sigma}}\left[\mathbf{e}^{p} \cdot \boldsymbol{\sigma}-\varphi_{\mathscr{E}}(\boldsymbol{\sigma})\right], \quad \varphi_{\mathscr{E}}(\boldsymbol{\sigma})= \begin{cases}0 & \text { if } \boldsymbol{\sigma} \in \mathscr{C}, \\ +\infty & \text { otherwise } .\end{cases}
$$

Due to Moreau's terminology [5] we call $\varphi_{\mathscr{E}}(\boldsymbol{\sigma})$ the plastic potential and $\varphi^{*}\left(\mathbf{e}^{p}\right)$ the dissipation function. Note that $\varphi^{*}\left(\mathbf{e}^{p}\right)$ is convex, lower-semicontinuous, subdifferentiable, and homogeneous of first degree:

$$
\varphi^{*}\left(\lambda \mathbf{e}^{p}\right)=\lambda \varphi^{*}\left(\mathbf{e}^{p}\right) \quad \text { for } \lambda \geq 0 .
$$

Applying the convex analysis to elastoplasticity Debordes [6] established the duality between (2) and (4) by showing that

$$
\alpha=\beta \text {. }
$$

The present paper is aimed at reducing the three-dimensional variational problems (2), (4) to the two-dimensional problems for the shakedown factor of elastoplastic shells. We call a shell approximation asymptotically exact if the calculated shakedown factor differs from the exact value (2) or (4) by a small quantity tending toward zero with the shell thickness. The approximation constructed in the present paper is valid for shell problems, where the elastic response $\underset{\sigma}{\mathrm{e}}$ corresponds to a membrane state [7]. Its realization requires a proper shell geometry or a strong restriction on the loading set. Nevertheless, this approximation allows for many interesting applications a further reduction of the two-dimensional variational problems to algebraic ones. This advantage of membrane approximation seems to be first noticed by Mosolov and Miasnikov [8] in their analysis of the limit load factor for rigid-plastic shells. The general theory accounting for the simultaneous effects of membrane and bending states of the elastic response lead to more complicated shell theories, in which either two-dimensionality or asymptotical exactness cannot be achieved (cf. Sawczuk [4], König [9], Weichert [10], Groß-Weege [11]).

In Sec. 2 the two-dimensional variational problems for the shakedown factor of the elastoplastic shell will be derived. The area of applicability and the restriction $o$ the loading set will be specified. In Sec. 3 the two-dimensional variational problem 
are reduced to an algebraic optimization procedure, which enables the determination of the shakedown factor analytically. Furthermore, in the case of nonalgebraization a calculation algorithm for the shakedown factor is proposed. For illustration, some examples are considered in Sec. 4. The last section is devoted to the proof of the asymptotic accuracy of the membrane approximation.

2. Shakedown factor for membrane approximation of elastoplastic shells. Let $\mathbf{g}_{a}$ and $g_{a b}$ be the natural base vectors and the components of the metric tensor associated with (1), respectively:

$$
\begin{array}{cll}
\mathbf{g}_{\alpha}=\mu_{\alpha}^{\beta} \mathbf{a}_{\beta}, & \mathbf{g}^{\alpha}=\left(\mu^{-1}\right)_{\beta}^{\alpha} \mathbf{a}^{\beta}, & \mathbf{g}_{3}=\mathbf{g}^{3}=\mathbf{n}, \\
g_{a b}=\mathbf{g}_{a} \cdot \mathbf{g}_{b}, & g^{a b}=\mathbf{g}^{a} \cdot \mathbf{g}^{b}, & a, b=1,2,3,
\end{array}
$$

where

$$
\mathbf{a}_{\alpha}=\mathbf{r}_{, \alpha}, \quad a_{\alpha \beta}=\mathbf{a}_{\alpha} \cdot \mathbf{a}_{\beta}, \quad \mathbf{a}^{\alpha}=a^{\alpha \beta} \mathbf{a}_{\beta}, \quad \alpha, \beta=1,2,
$$

are the same base vectors and the metric tensor of the smooth surface $\Omega$ and

$$
\begin{gathered}
u_{\alpha}^{\beta}=\delta_{\alpha}^{\beta}-\xi b_{\alpha}^{\beta}, \quad\left(\mu^{-1}\right)_{\alpha}^{\beta}=\frac{1}{\mu}\left[\delta_{\alpha}^{\beta}-\xi\left(2 H \delta_{\alpha}^{\beta}-b_{\alpha}^{\beta}\right)\right], \\
\mu=\operatorname{det} \mu_{\alpha}^{\beta}=1-2 H \xi+K \xi^{2}, \\
b_{\alpha \beta}=\mathbf{a}_{\alpha, \beta} \cdot \mathbf{n}, \quad H=\frac{1}{2} b_{\alpha}^{\alpha}, \quad K=\operatorname{det} b_{\alpha}^{\beta} .
\end{gathered}
$$

Let homogeneous kinematical boundary conditions be specified on one part of the shell edge, and statical boundary conditions on the facial surfaces and on the remaining part of the shell edge:

$$
\begin{aligned}
& \mathscr{B}_{T}=\Omega^{+} \cup \Omega^{-} \cup\left(\Gamma_{T} \times(-h / 2, h / 2)\right), \\
& \mathscr{B}_{x}=\Gamma_{x} \times(-h / 2, h / 2), \quad \overline{\Gamma_{x} \cup \Gamma_{T}}=\Gamma, \\
& \Omega^{ \pm}=\left\{\mathbf{X}\left(\xi^{\alpha}, \xi\right) \mid \xi= \pm h / 2\right\} .
\end{aligned}
$$

With respect to the coordinates $\xi^{\alpha}, \xi$ the equations (3) can be rewritten as follows (see, for example, [12]):

$$
\begin{aligned}
& \tau_{\mid \beta}^{\alpha \beta}+\frac{\partial}{\partial \xi}\left(\mu_{\beta}^{\alpha} \tau^{\beta}\right)-\tau^{\beta} b_{\beta}^{\alpha}=0, \\
& \tau_{\mid \beta}^{\beta}+\tau^{\alpha \beta} b_{\alpha \beta}+\frac{\partial}{\partial \xi} \tau=0, \\
& \left.\tau^{\alpha}\right|_{\xi= \pm h / 2}=0,\left.\quad \tau\right|_{\xi= \pm h / 2}=0,
\end{aligned}
$$

where $\tau^{\alpha \beta}=\mu_{\lambda}^{\alpha} \rho^{\lambda \beta} \mu, \tau^{\alpha}=\rho^{\alpha 3} \mu$, and $\tau=\rho^{33} \mu$. Throughout the text the symbol $(\cdot)_{\mid \alpha}$ indicates covariant differentiation based on a connection associated with the metric $a_{\alpha \beta}$ of the middle surface $\Omega$.

In order to simplify the variational problem (2) we shall assume that the elastic response $\stackrel{\mathrm{e}}{\sigma}$ corresponds to the membrane state of the shell [7],

$$
\stackrel{\mathrm{e}}{\sigma} \alpha \beta=\frac{1}{h} \stackrel{\mathrm{e}}{n}^{\alpha \beta}\left(\xi^{\alpha}\right)+O\left(\frac{h}{R}\right) \frac{\mathrm{e}}{h}, \quad \mathrm{e}^{\alpha} \alpha 3=O\left(\frac{h}{R}\right) \stackrel{\mathrm{e}}{h}, \quad \stackrel{\mathrm{e}}{\sigma} 33=O\left(\frac{h}{R}\right) \frac{\mathrm{e}}{h},
$$


where $\stackrel{\mathrm{e}}{\mathrm{e}}^{\alpha \beta}$ is the stress resultant tensor, $R$ is a characteristic radius of curvature of the middle surface $\Omega$, and

$$
\stackrel{\mathrm{e}}{n}=\max _{\xi^{\prime \prime}, \alpha, \beta}\left\{\mathrm{e}^{\alpha \beta}\left(\xi^{\alpha}\right)\right\} .
$$

We assume also that the characteristic scale $L$ of the variation of deformation pattern [12] is of the same order as $R$. Then the stress resultant tensor for the isotropically elastic comparison shell should satisfy the so-called membrane shell equations [7, 12]

$$
\begin{aligned}
& \stackrel{\mathrm{e}}{n}{ }_{\mid \beta}^{\alpha \beta}+p^{\alpha}=0, \quad \stackrel{\mathrm{e}}{n} \alpha \beta b_{\alpha \beta}+p=0, \\
& \stackrel{\mathrm{e}}{n}^{\alpha \beta} \nu_{\beta}=q^{\alpha} \quad \text { on } \Gamma_{T}, \quad \stackrel{\mathrm{e}}{u}_{\alpha}=0 \quad \text { on } \Gamma_{x}, \\
& \stackrel{\mathrm{e}}{n}^{\alpha \beta}=\frac{E h}{1-\nu^{2}}\left[\nu \gamma_{\lambda}^{\lambda} a^{\alpha \beta}+(1-\nu) \gamma^{\alpha \beta}\right] \text {, } \\
& \gamma_{\alpha \beta}=\frac{1}{2}\left(\stackrel{e}{u}_{\alpha \mid \beta}+\stackrel{e}{u}_{\beta \mid \alpha}\right)-b_{\alpha \beta} \stackrel{e}{u} \text {, }
\end{aligned}
$$

where $E$ is Young's modulus and $\nu$ is the Poisson ratio. For statically determinate problems only the first three equations of (12) need to be solved in order to define $\mathrm{e}^{\alpha \beta}$.

The membrane state of elastic response can be realized in shells of positive Gaussian curvature (except for a small edge layer). For shells of zero or negative curvature, the stress state (11) may still be realized, if a certain orthogonality condition is imposed on the external loads $[7,8]$

$$
\int_{\Omega}\left(p^{\alpha} \stackrel{\circ}{u}_{\alpha}+p \stackrel{\circ}{u}\right) d A+\int_{\Gamma_{T}} q^{\alpha} \stackrel{\circ}{u}_{\alpha} d s=0
$$

with $d A$ the surface element. Here the displacement field $\stackrel{\circ}{u}_{\alpha}, \stackrel{\circ}{u}$ describes the deformation of the middle surface with vanishing strain (the analogy to the rigid deformation of three-dimensional bodies):

$$
\frac{1}{2}\left(\stackrel{\circ}{u}_{\alpha \mid \beta}+\stackrel{\circ}{u}_{\beta \mid \alpha}\right)-b_{\alpha \beta} \stackrel{\circ}{u}=0 .
$$

Condition (13) restricts considerably the area of applicability of membrane approximation for shells of zero or negative curvature.

Assuming the validity of (11) let us choose the following self-equilibrated residual stress field:

$$
\rho^{\alpha \beta}=\frac{1}{h} s^{\alpha \beta}\left(\xi^{\alpha}\right), \quad \rho^{\alpha 3}=O\left(\frac{h}{R}\right) \frac{s}{h}, \quad \rho^{33}=O\left(\frac{h}{R}\right) \frac{s}{h}
$$

with $s=\max \left\{s^{\alpha \beta}\right\}$. Here the residual stress resultant tensor $s^{\alpha \beta}$ has to satisfy the equations

$$
s_{\mid \beta}^{\alpha \beta}=0, s^{\alpha \beta} b_{\alpha \beta}=0 \quad \text { in } \Omega, \quad s^{\alpha \beta} \nu_{\beta}=0 \quad \text { on } \Gamma_{T} .
$$

In the last section it will be shown that self-equilibrated residual stress fields of the type (15) exist. Let the space of all time-independent $s^{\alpha \beta}\left(\xi^{\alpha}\right)$ satisfying the equations (16) be denoted by $\mathscr{J}_{2}$. We then reduce the variational problem (2) to

$$
\begin{array}{r}
\alpha_{2}=\sup \left\{v \mid v \in \mathbb{R}, \exists s^{\alpha \beta} \in \mathscr{J}_{2}, \forall t \in[0, T]\right. \\
\left.v\left(\stackrel{\mathrm{e}}{n}^{\alpha \beta}+s^{\alpha \beta}\right) \in \mathscr{C}_{2}\right\} .
\end{array}
$$


Here $\mathscr{C}_{2}$ is the set of $n^{\alpha \beta}=\stackrel{\mathrm{e}}{n}^{\alpha \beta}+s^{\alpha \beta}$ such that

$$
\left(\sigma^{\alpha \beta}, \sigma^{\alpha 3}, \sigma^{33}\right)=\left(\frac{n^{\alpha \beta}}{h}+O\left(\frac{h}{R}\right) \frac{n}{h}, O\left(\frac{h}{R}\right) \frac{n}{h}, O\left(\frac{h}{R}\right) \frac{n}{h}\right) \in \mathscr{C}
$$

with $n=\max \left\{n^{\alpha \beta}\right\}$. If, for example, the Mises yield condition is postulated, then the set $\mathscr{C}$ is given by the inequality

$$
\left[\left(g_{a c} g_{b d}-\frac{1}{3} g_{a b} g_{c d}\right) \sigma^{a b} \sigma^{c d}\right]^{1 / 2} \leq \sigma_{0} \sqrt{\frac{2}{3}}
$$

with $\sigma_{0}$ the yield stress defined by the uni-axial tension experiment. Because of the smallness of $h / R$ we can neglect in (18) and (19) all terms of order $h / R$ in comparison with unity and specify $\mathscr{C}_{2}$ by

$$
\left[\left(a_{\alpha \gamma} a_{\beta \delta}-\frac{1}{3} a_{\alpha \beta} a_{\gamma \delta}\right) n^{\alpha \beta} n^{\gamma \delta}\right]^{1 / 2} \leq h \sigma_{0} \sqrt{\frac{2}{3}} .
$$

It is obvious from (20) that $\mathscr{C}_{2}$ is a convex set in the space of all symmetric tensor fields $n^{\alpha \beta}$. The variational problem (17), (16), (20) determines the statical shakedown factor for the membrane approximation.

To simplify the variational problem (4) for the kinematical shakedown factor we rewrite it in the form

$$
\begin{gathered}
\beta=\inf \int_{0}^{T} \int_{-h / 2}^{h / 2} \int_{\Omega} \varphi^{*}\left(\mathbf{e}^{p}\right) \mu d A d \xi d t \\
\int_{0}^{T} \mathbf{e}^{p} d t=\varepsilon_{a b}^{p} \mathbf{g}^{a} \otimes \mathbf{g}^{b}, \quad \varepsilon_{\alpha \beta}^{p}=\frac{1}{2}\left(\mu_{\alpha}^{\lambda} \mathbf{a}_{\lambda} \cdot \mathbf{w}_{, \beta}+\mu_{\beta}^{\lambda} \mathbf{a}_{\lambda} \cdot \mathbf{w}_{, \alpha}\right), \\
\varepsilon_{\alpha 3}^{p}=\frac{1}{2}\left(\mu_{\alpha}^{\lambda} \mathbf{a}_{\lambda} \cdot \mathbf{w}_{, \xi}+\mathbf{n} \cdot \mathbf{w}_{, \alpha}\right), \quad \varepsilon_{33}^{p}=\mathbf{n} \cdot \mathbf{w}_{, \xi}, \\
\int_{0}^{T} \int_{-h / 2}^{h / 2} \int_{\Omega}\left(\stackrel{\mathrm{e}}{\sigma}^{\alpha \beta} e_{\alpha \beta}^{p}+2 \stackrel{\mathrm{e}}{\sigma}^{\alpha 3} e_{\alpha 3}^{p}+\stackrel{\mathrm{e}}{\sigma}^{33} e_{33}^{p}\right) \mu d A d \xi d t=1 .
\end{gathered}
$$

Having specified $\mathscr{C}$ by (19) we obtain the following formula for $\varphi^{*}\left(\mathbf{e}^{p}\right)$ :

$$
\varphi^{*}\left(\mathbf{e}^{p}\right)=\left\{\begin{array}{l}
\sigma_{0} \sqrt{\frac{2}{3}}\left[g^{a b} g^{c d} e_{a b}^{p} e_{c d}^{p}\right]^{1 / 2} \\
+\infty \quad \text { if } g^{a b} e_{a b}^{p}=0,
\end{array}\right.
$$

The last condition of (22) means that only the incompressible tensor fields $\mathbf{e}^{p}$ can be actually realized in the elastoplastic body.

Let us choose the following "trial" plastic strain rate fields:

$$
e_{\alpha \beta}^{p}=f_{\alpha \beta}\left(\xi^{\alpha}, t\right)+O\left(\frac{h}{R}\right) f, \quad e_{\alpha 3}^{p}=O\left(\frac{h}{R}\right) f, \quad e_{33}^{p}=-a^{\alpha \beta} f_{\alpha \beta}\left(\xi^{\alpha}, t\right)+O\left(\frac{h}{R}\right) f,
$$

where $f=\max _{\xi^{\prime \prime}, \alpha, \beta}\left\{f_{\alpha \beta}\left(\xi^{\alpha}, t\right)\right\}$. The tensor $f_{\alpha \beta}$ is chosen to satisfy the compatibility condition

$$
\begin{gathered}
A_{\alpha \beta}=\int_{0}^{T} f_{\alpha \beta} d t=\frac{1}{2}\left(u_{\alpha \mid \beta}+u_{\beta \mid \alpha}\right)-b_{\alpha \beta} u, \\
u_{\alpha}=0 \quad \text { on } \Gamma_{x},
\end{gathered}
$$


where $u_{\alpha}, u$ is some displacement field of the middle surface. In the last section it will be shown that the plastic strain rate field of (23) exactly satisfying the threedimensional compatibility and incompresibility conditions exists.

Substituting the fields (23) into the functional $(21)_{1}$ and using the assumption (11) to neglect all terms of order $O(h / R)$ in comparison with unity, we obtain the following two-dimensional variational problem:

$$
\beta_{2}=\inf \left\{\int_{0}^{T} \int_{\Omega} \varphi^{*}\left(f_{\alpha \beta}\right) d A d t \mid \int_{0}^{T} f_{\alpha \beta} d t \in \mathscr{R}_{2}, \int_{0}^{T} \int_{\Omega} \frac{1}{h} n^{\alpha \beta} f_{\alpha \beta} d A d t=1\right\} .
$$

Here $\mathscr{K}_{2}$ is used to denote the set of all strain fields of (24). The dissipation function $\varphi^{*}\left(f_{\alpha \beta}\right)$ is given by

$$
\varphi_{2}^{*}\left(f_{\alpha \beta}\right)=\sigma_{0} \sqrt{\frac{2}{3}}\left[\left(a^{\alpha \gamma} a^{\beta \delta}+a^{\alpha \beta} a^{\gamma \delta}\right) f_{\alpha \beta} f_{\gamma \delta}\right]^{1 / 2} .
$$

One can easily show that

$$
\varphi_{2}^{*}\left(f_{\alpha \beta}\right)=\max _{n^{\alpha \beta}}\left[\frac{1}{h} f_{\alpha \beta} n^{\alpha \beta}-\varphi_{\mathscr{C}_{2}}\left(n^{\alpha \beta}\right)\right],
$$

where $\varphi_{\mathscr{C}_{2}}\left(n_{\alpha \beta}\right)$ is the indicator function of the set $\mathscr{C}_{2}$. Therefore by applying the standard method of convex analysis [6] one can establish the duality between (17) and (25) yielding

$$
\alpha_{2}=\beta_{2} .
$$

3. Calculation of the shakedown factor. Due to the equality (27) we can restrict ourselves to the variational problem (25) for the kinematical shakedown factor. Let us rewrite $(25)$ in the form

$$
\beta_{2}^{-1}=\sup _{f_{\alpha \beta} \in \mathscr{F}_{2}}\left[\int_{0}^{T} \int_{\Omega} \frac{1}{h} \stackrel{\mathrm{e}}{n}^{\alpha \beta} f_{\alpha \beta} d A d t / \int_{0}^{T} \int_{\Omega} \varphi^{*}\left(f_{\alpha \beta}\right) d A d t\right] .
$$

Suppose that the equations (24) have at least one solution for every given symmetric tensor $A_{\alpha \beta}=\int_{0}^{T} f_{\alpha \beta} d t$. The analysis of (24) is fully presented in [13]. In this case we can drop the constraint (24) in the variational problem (28) and write

$$
\begin{aligned}
\beta_{2}^{-1} & =\sup _{f_{\alpha \beta}\left(\xi^{\alpha}, t\right)}\left[\int_{0}^{T} \int_{\Omega} \frac{1}{h} \stackrel{\mathrm{e}}{n}^{\alpha \beta} f_{\alpha \beta} d A d t / \int_{0}^{T} \int_{\Omega} \varphi_{2}^{*}\left(f_{\alpha \beta}\right) d A d t\right] \\
& =\sup _{\xi^{\alpha}, t, f_{\alpha \beta}}\left[\frac{1}{h} \stackrel{\mathrm{e}}{ }^{\alpha \beta} f_{\alpha \beta} / \varphi_{2}^{*}\left(f_{\alpha \beta}\right)\right] .
\end{aligned}
$$

The theorem on the norm of a linear continuous functional in $L_{1}(\Omega \times[0, T])$ is used in obtaining (29) (cf. also [8]). According to (29) the calculation of the shakedown factor within the membrane approximation is reduced to an algebraic problem of optimizing the function of six variables $\xi^{\alpha}, t, f_{\alpha \beta}$. Corresponding results had been obtained by Mosolov and Miasnikov [8] for the limit load factor of rigid-plastic shells. When $\varphi_{2}^{*}\left(\xi^{\alpha}, f_{\alpha \beta}\right)$ is a smooth function of the variables $f_{\alpha \beta}$, the extremal tensor $\bar{f}_{\alpha \beta}$ is determined by the equations

$$
\frac{\partial \varphi_{2}^{*}}{\partial f_{\alpha \beta}}=\frac{1}{h} \stackrel{\mathrm{e}}{\alpha \beta}^{\alpha \beta}
$$


and the shakedown factor can be found by

$$
\beta_{2}^{-1}=\sqrt{\frac{3}{2}} \frac{1}{h \sigma_{0}} \max _{\xi^{\alpha}, t} \sqrt{\left(a_{\alpha \gamma} a_{\beta \delta}-\frac{1}{3} a_{\alpha \beta} a_{\gamma \delta}\right) n^{\mathrm{e} \alpha \beta} n^{\mathrm{e} \nu \delta}} .
$$

If some kinematical constraints of integral type are additionally imposed on the admissible fields $u_{\alpha}$, then the theorem on the norm of a linear functional in $L_{1}(\Omega \times[0, T])$ cannot be directly used to obtain (29). However, we can apply the algorithm based on the Lagrange multiplier method [8]. Let a linear continuous functional $F(f)$ be defined on a subspace $\mathbb{M}$ of the Banach space $\mathbb{B}$, extracted by a finite system of linear continuous functionals $T_{i}(f), T_{i}(f)=0, i=1, \ldots, N$. Then

$$
\|F\|_{\mathbb{M}}=\inf _{\lambda_{i}} \sup _{f \in \mathbb{B}}\left\{\left[F(f)-\sum_{i=1}^{N} \lambda_{i} T_{i}(f)\right] /\|f\|_{\mathbb{B}}\right\} .
$$

4. Analytical examples. We suppose the Mises yield condition (19) to be the case in all examples considered in this section.

EXAMPLE 1. Let us examine a spherical shell of radius $R$ under an internal pressure $p(t), 0 \leq p(t) \leq p^{+}$. The membrane equations have the form

$$
\stackrel{\mathrm{e}}{n}_{\mid \beta}^{\alpha \beta}=0, \quad \stackrel{\mathrm{e}}{n}^{\alpha \beta} b_{\alpha \beta}+p=0, \quad b_{\alpha \beta}=-\frac{1}{R} a_{\alpha \beta} .
$$

The elastic response is equal to

$$
\mathrm{e}^{\alpha \beta}=p(t) R a^{\alpha \beta}
$$

and results in the shakedown factor $\beta_{2}=h \sigma_{0} / p^{+} R$.

EXAMPLE 2. Let a long cylindrical shell of length $L$ with circular cross section of radius $R \ll L$ be subjected to the normal pressure $p(t)=p^{+} v_{1}(t), 0 \leq v_{1}(t) \leq 1$, and the axial forces $q(t)=q^{+} v_{2}(t), 0 \leq v_{2}(t) \leq 1$, on the edges $\xi^{2}=0, L$ (Fig. 1 ). It is easy to see that the orthogonality condition (13) for such types of force is satisfied. The elastic response is found to be of the form

$$
\stackrel{\mathrm{e}}{n}^{11}=-R p(t), \quad \stackrel{e}{n} 22=-q(t) .
$$
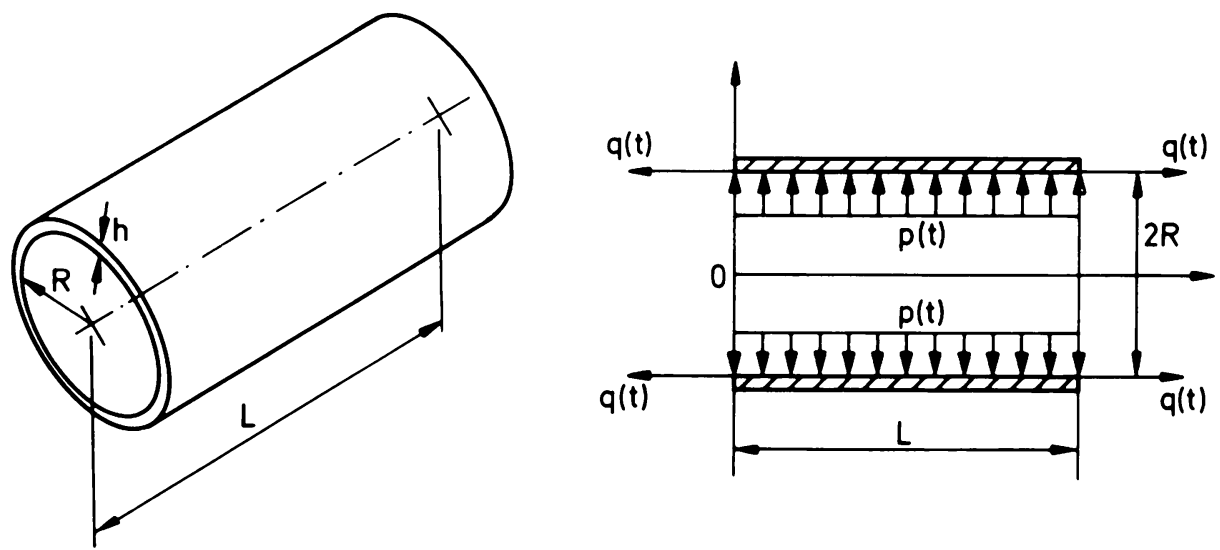

FIG. 1 


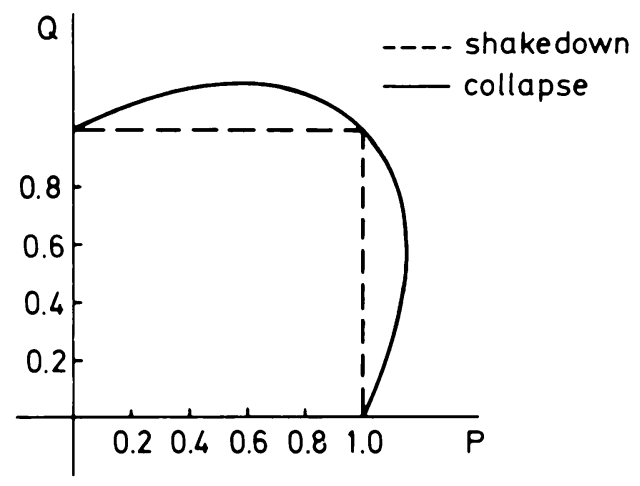

FIG. 2

With

$$
a_{11}=1, \quad a_{22}=1, \quad a_{12}=0, \quad P^{+}=\frac{p^{+} R}{h \sigma_{0}}, \quad Q^{+}=\frac{q^{+}}{h \sigma_{0}}
$$

the shakedown factor is expressed by

$$
\beta_{2}^{-1}=\max _{t}\left(P^{+2} v_{1}^{2}+Q^{+2} v_{2}^{2}-P^{+} Q^{+} v_{1} v_{2}\right)^{1 / 2} .
$$

Instead of calculating the shakedown factor for the specific loading path $v_{1}(t), v_{2}(t)$, one can consider all possible paths and calculate the smallest shakedown factor. This leads to the problem

$$
\beta_{2}^{-1}=\max _{v_{1}, v_{2}}\left(P^{+2} v_{1}^{2}+Q^{+2} v_{2}^{2}-P^{+} Q^{+} v_{1} v_{2}\right)^{1 / 2} .
$$

The shakedown diagram of (33) is shown in Fig. 2 by the curve 2 . The curve 1 margins the adaptation region with respect to proportional loading $\left(v_{1}=v_{2}\right)$.

EXAMPle 3. Consider a torus shell (Fig. 3)

$$
\begin{aligned}
& x^{1}=\left[b+a \cos \left(\xi^{1} / a\right)\right] \cos \xi^{2}, \\
& x^{2}=\left[b+a \cos \left(\xi^{1} / a\right)\right] \sin \xi^{2}, \\
& x^{3}=\left[a \sin \left(\xi^{1} / a\right)\right],
\end{aligned}
$$

subjected to a variable pressure $p(t)=p^{+} v_{1}(t), 0 \leq v_{1}(t) \leq 1$, and a centrifugal force with components

$$
\begin{gathered}
F_{1}=q(t)\left[b+a \cos \left(\xi^{1} / a\right)\right] \cos \xi^{2}, \quad F_{2}=q(t)\left[b+a \cos \left(\xi^{1} / a\right)\right] \sin \xi^{2}, \quad F_{3}=0, \\
q(t)=q^{+} v_{2}(t), \quad 0 \leq v_{2}(t) \leq 1 .
\end{gathered}
$$

The shell geometry is characterized by

$$
\begin{gathered}
a_{11}=A_{1}^{2}=1, \quad a_{12}=0, \quad a_{22}=A_{2}^{2}=\left[b+a \cos \left(\xi^{1} / a\right)\right]^{2}, \\
b_{11}=-\frac{A_{1}^{2}}{R_{1}}=-\frac{1}{a}, \quad b_{12}=0, \quad b_{22}=-\frac{A_{2}^{2}}{R_{2}}=\left[b+a \cos \left(\xi^{1} / a\right)\right] \cos \left(\xi^{1} / a\right) .
\end{gathered}
$$




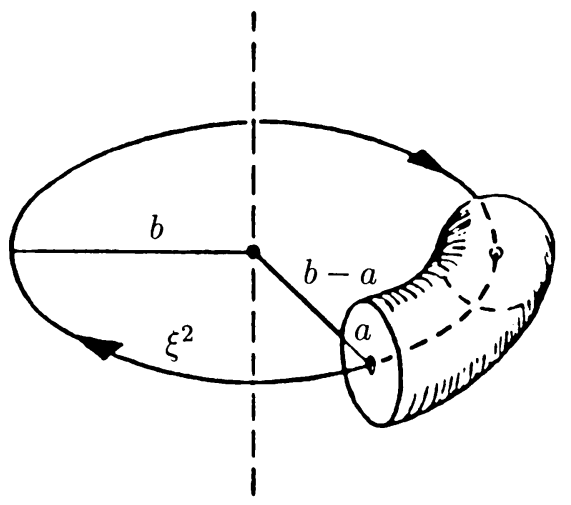

Fig. 3. Torus.

The equations (14) for infinitesimal flexures take the form [7]

$$
\begin{aligned}
& \frac{1}{A_{1}} \stackrel{\circ}{v}_{1,1}+\frac{A_{1,2}}{A_{1} A_{2}} \stackrel{\circ}{2}_{2}+\frac{1}{R_{1}} \stackrel{\circ}{v}=0, \\
& \frac{1}{A_{2}} \stackrel{\circ}{v}_{2,2}+\frac{A_{2,1}}{A_{1} A_{2}} \stackrel{\circ}{v}_{1}+\frac{1}{R_{2}} \stackrel{\circ}{v}=0, \\
& \left(\frac{\stackrel{\circ}{v}_{1}}{A_{1}}\right)_{, 2} \frac{A_{1}}{A_{2}}+\left(\frac{\stackrel{\circ}{v}_{2}}{A_{2}}\right), 1 \frac{A_{2}}{A_{1}}=0,
\end{aligned}
$$

where $\stackrel{\circ}{v}_{1}, \stackrel{\circ}{v}_{2}$, and $\stackrel{\circ}{v}$ are physical components of the displacement vector $\stackrel{\circ}{u}_{\alpha}, \stackrel{\circ}{u}$. The following nontrivial solution of (34) exists:

$$
\begin{aligned}
& \stackrel{\circ}{v}_{1}= \begin{cases}C_{1} \cos \left(\xi^{1} / a\right), & \left|\xi^{1}\right| \leq \pi a / 2, \\
C_{2} \cos \left(\xi^{1} / a\right), & \pi a / 2 \leq\left|\xi^{1}\right| \leq \pi a,\end{cases} \\
& \stackrel{\circ}{v}_{2}=0, \quad \stackrel{\circ}{v}= \begin{cases}C_{1} \sin \left(\xi^{1} / a\right), & \left|\xi^{1}\right| \leq \pi a / 2, \\
C_{2} \sin \left(\xi^{1} / a\right), & \pi a / 2 \leq\left|\xi^{1}\right| \leq \pi a .\end{cases}
\end{aligned}
$$

It is easy to check the orthogonality condition (13) with respect to this flexure for the given force. Solving Eq. (12) written in the orthogonal coordinate system $\xi^{1}, \xi^{2}$, one finds the physical components of $n^{\mathrm{e}} \alpha \beta$ to be

$$
\begin{aligned}
& N_{11}=\frac{p(t) a\left[2 b+a \cos \left(\xi^{1} / a\right)\right]}{2\left[b+a \cos \left(\xi^{1} / a\right)\right]}, \quad N_{12}=0, \\
& N_{22}=q(t)\left[b+a \cos \left(\xi^{1} / a\right)\right]^{2}+p(t) a / 2 .
\end{aligned}
$$

We return now to the optimization problem (29). Introducing the notation

$$
\begin{aligned}
& N_{1}=P^{+} v_{1} \frac{2 b / a+\cos \left(\xi^{1} / a\right)}{2\left[b / a+\cos \left(\xi^{1} / a\right)\right]}, \\
& N_{2}=Q^{+} v_{2}\left[b / a+\cos \left(\xi^{1} / a\right)\right]^{2}+P^{+} v_{1} / 2, \quad P^{+}=\frac{p^{+} a}{h \sigma_{0}}, Q^{+}=\frac{q^{+} a^{2}}{h \sigma_{0}},
\end{aligned}
$$




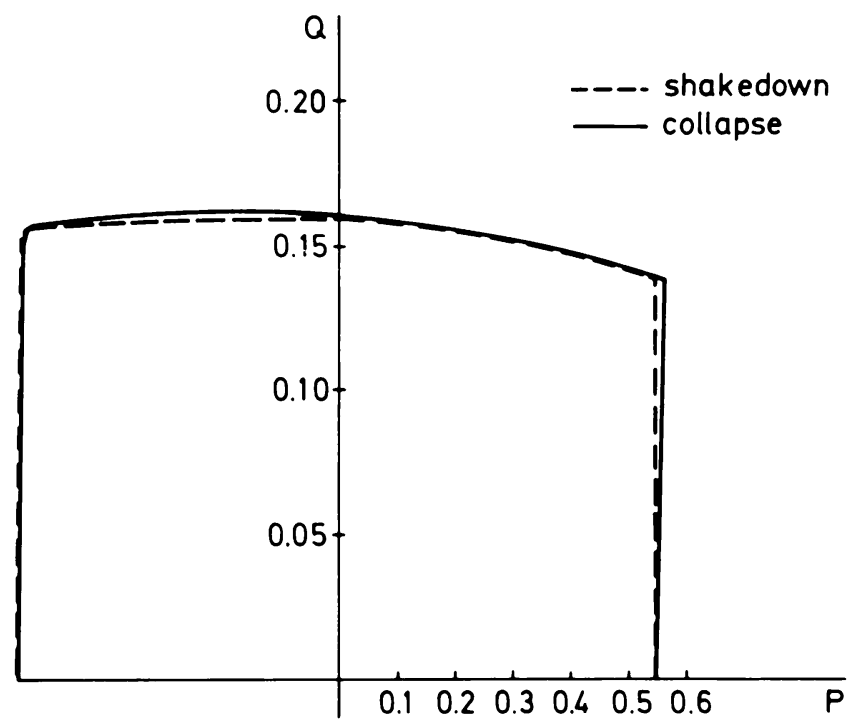

FIG. 4

and calculating the smallest $\beta_{2}$ for all possible paths, one arrives at

$$
\beta_{2}^{-1}=\max _{\xi^{1}, v_{1}, v_{2}}\left(N_{1}^{2}+N_{2}^{2}-N_{1} N_{2}\right)^{1 / 2} .
$$

The shakedown diagrams of (35) with $b / a=1.5$ are presented in Fig. 4. Two different cases are considered: (a) $P^{+}>0$ (interior pressure), $Q^{+}>0$ and (b) $P^{+}<0$ (exterior pressure), $Q^{+}>0$. The curve 1 (2) margins the adaptation region with respect to proportional (two-parameter) loadings.

EXAMPLE 4. Let us consider a long cylindrical shell $(R \ll L)$ subjected to the uniform pressure $p(t)=p^{R}+p^{r} v(t), 0 \leq v(t) \leq 1, p^{R}=$ const. If the shell is fixed only at the edge $\xi^{2}=0$, then the shakedown factor is equal to $\beta_{2}=\left(P^{R}+P^{r}\right)^{-1}$, where $P^{R}=p^{R} R / h \sigma_{0}$ and $P^{r}=p^{r} R / h \sigma_{0}$. Now we examine the shell adaptation under the additional boundary conditions $u_{1}(L)=u_{2}(L)=0$. These conditions result in the following constraint for $f_{22}$ :

$$
\int_{0}^{T} \int_{0}^{L} f_{22} d \xi^{2} d t=0
$$

By using (32) we obtain for $\beta_{2}$

$$
\beta_{2}^{-1} \min _{\lambda} \max _{v}\left[\left(P^{R}+P^{r} v\right)^{2}+\lambda^{2}-\left(P^{R}+P^{r} v\right) \lambda\right]^{1 / 2}=\frac{\sqrt{3}}{2}\left(P^{R}+P^{r}\right) .
$$

The shakedown diagram of (36) is presented in Fig. 5. The curve 1 (2) margins the adaptation region with the one (two) fixed shell edge(s). One can see that fixing the econd edge of the shell results in an increase of the shakedown region. 


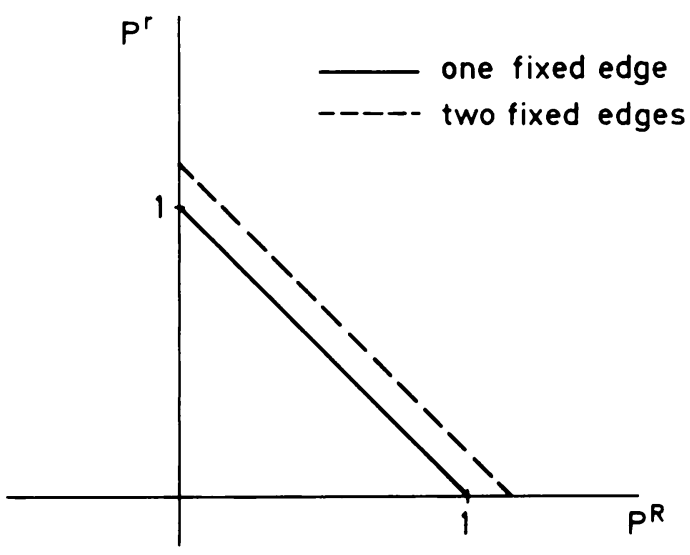

FIG. 5

5. Asymptotic accuracy of the membrane shell approximation for the shakedown theory. We shall prove the asymptotic accuracy of the membrane approximation assuming for simplicity that the membrane state of elastic response is realized everywhere in the shell (as in the case of closed shells). The more subtle case should be analyzed with the help of a boundary layer (see, for example, [8]). The idea of the proof is to determine the upper and lower bounds, the difference of which should tend toward zero with the shell thickness.

(a) Self-equilibrated residual stress field $\boldsymbol{\rho}_{h}$. Let us determine the components $\rho_{h}^{\alpha \beta}$ of the residual stress field in the form

$$
\rho_{h}^{\alpha \beta}=\frac{1}{h} s^{\alpha \beta}\left(\xi^{\alpha}\right)
$$

where $s^{\alpha \beta}$ satisfies (16). Solving the exact three-dimensional equations (10) as a system of ordinary differential equations with unknown functions $\rho_{h}^{\alpha 3}$ and $\rho_{h}^{33}$, we find the remaining components of the self-equilibrated stress field $\boldsymbol{\rho}_{h}$. It turns out that (16) are sufficient conditions for the existence of $\rho_{h}^{\alpha 3}$ and $\rho_{h}^{33}$. Now from (2) it follows that

$$
\alpha \geq \alpha_{2}=\sup \left\{v \mid v \in \mathbb{R}, \exists s^{\alpha \beta} \in \mathscr{F}_{2}, \forall t \in[0, T] v\left(\stackrel{\mathrm{e}}{\boldsymbol{\sigma}}+\boldsymbol{\rho}_{h}\right) \in \mathscr{C}\right\} .
$$

Analyzing (10) one can see that

$$
\rho_{h}^{\alpha 3}=O\left(\frac{h}{R}\right) \frac{s}{h}, \quad \rho_{h}^{33}=O\left(\frac{h}{R}\right) \frac{s}{h} .
$$

Modifying the parameter $v$ on a small quantity $\varepsilon$ one can show that two conditions

$$
\mu\left(\stackrel{\mathrm{e}}{\boldsymbol{\sigma}}+\boldsymbol{\rho}_{h}\right) \in \mathscr{C} \quad \text { and } \quad \mu(1+\varepsilon)\left(\stackrel{\mathrm{e}}{n}^{\alpha \beta}+s^{\alpha \beta}\right) \in \mathscr{C}_{2}
$$

are equivalent (cf. (11), (39) and the formulae (8), (9)). This means that

$$
\alpha_{2}^{h}=\alpha_{2}+\gamma(h), \quad \gamma(h) \rightarrow 0 \text { for } h \rightarrow 0,
$$

where $\alpha_{2}$ is the solution of (17). Therefore

$$
\alpha \geq \alpha_{2}+\gamma(h), \quad \gamma(h) \rightarrow 0 \text { for } h \rightarrow 0 .
$$


(b) Kinematically admissible strain rate field $\mathbf{e}_{h}^{p}$. For every given tensor $f_{\alpha \beta}$ let us solve the following problem:

$$
\frac{1}{2}\left(v_{\alpha \mid \beta}+v_{\beta \mid \alpha}\right)-b_{\alpha \beta} v=f_{\alpha \beta}, \quad v_{\alpha}=0 \quad \text { on } \Gamma_{x} .
$$

Having found $v_{\alpha}, v$ we construct the modified Kichhoff-Love velocity field

$$
\begin{aligned}
& \dot{w}_{\alpha}=\mathbf{a}_{\alpha} \cdot \dot{\mathbf{w}}=v_{\alpha}-\xi \psi_{\alpha}, \\
& \dot{w}=\mathbf{n} \cdot \dot{\mathbf{w}}=v+h y\left(\xi^{\alpha}, \xi, t\right), \\
& \psi_{\alpha}=v_{\mid \alpha}+b_{\alpha}^{\beta} v_{\beta}
\end{aligned}
$$

with the unknown function $y\left(\xi^{\alpha}, \xi, t\right)$. According to (21) the compatible strain rate field $\mathbf{e}_{h}^{p}$ is calculated by

$$
\begin{aligned}
& e_{\alpha \beta h}^{p}=\frac{1}{2}\left(\mu_{\alpha}^{\lambda} v_{\lambda \mid \beta}-\mu_{\alpha}^{\lambda} \xi \psi_{\lambda \mid \beta}+\mu_{\beta}^{\lambda} v_{\lambda \mid \alpha}-\mu_{\beta}^{\lambda} \xi \psi_{\lambda \mid \alpha}-2 \mu_{\alpha}^{\lambda} b_{\lambda \beta} v-2 \mu_{\alpha}^{\lambda} b_{\lambda \beta} h y\right), \\
& e_{\alpha 3 h}^{p}=\frac{1}{2}\left(-\mu_{\alpha}^{\lambda} \psi_{\lambda}+v_{\mid \alpha}+h y_{\mid \alpha}+b_{\alpha}^{\lambda} v_{\lambda}-b_{\alpha}^{\lambda} \xi \psi_{\lambda}\right), \\
& e_{33 h}^{p}=h y_{, \xi} .
\end{aligned}
$$

We choose the function $y$ so that the incompressibility condition $g^{a b} e_{a b h}^{p}=0$ is exactly satisfied. This leads to the following ordinary differential equation with respect to the unknown function $y$ :

$$
\begin{gathered}
h y_{, \xi}+g^{\alpha \beta} e_{\alpha \beta h}^{p}=0, \\
g^{\alpha \beta}=\frac{1}{\kappa^{2}}\left[(1-2 H \xi) a^{\alpha \beta}+2 \xi(1-2 H \xi) b^{\alpha \beta}+\xi^{2} b^{\alpha \lambda} b_{\lambda}^{\beta}\right],
\end{gathered}
$$

with $e_{\alpha \beta h}^{p}$ due to (45). Having solved (46) we can define all components of the plastic strain rate field (45). Because of the kinematical admissibility of the strain rate field (45), we can conclude with (11) that

$$
\beta_{2}^{-1} \leq \beta_{2}^{h-1}=\sup \left[\int_{0}^{T} \int_{\mathscr{B}} \frac{1}{h} \stackrel{\mathrm{e}}{\boldsymbol{\sigma}} \cdot \mathbf{e}_{h}^{p} d X d t / \int_{0}^{T} \int_{\mathscr{B}} \varphi_{2}^{*}\left(\mathbf{e}_{h}^{p}\right) d X d t\right]
$$

with $\mathbf{e}_{h}^{p}$ according to (45). Analyzing (45) and (46) one arrives at the following asymptotic formulae:

$$
\begin{aligned}
& e_{\alpha \beta h}^{p}=f_{\alpha \beta}\left(\xi^{\alpha}, t\right)+O\left(\frac{h}{R}\right) f, \\
& e_{\alpha 3 h}^{p}=O\left(\frac{h}{R}\right) f, \\
& e_{33 h}^{p}=-a^{\alpha \beta} f_{\alpha \beta}\left(\xi^{\alpha}, t\right)+O\left(\frac{h}{R}\right) f .
\end{aligned}
$$

From (11), (47), (48) it follows that

$$
\beta_{2}^{h}=\beta_{2}+\delta(h), \quad \delta(h) \rightarrow 0 \text { for } h \rightarrow 0,
$$

where $\beta_{2}$ is the solution of (25). The asymptotic accuracy of the membrane shell approximation for the shakedown factor follows then by (42), (49), (7), and (27). 


\section{REFERENCES}

[1] E. Melan, Theorie statisch unbestimmter Tragwerke aus ideal-plastischem Baustoff, Sitzungsber. Akad. Wiss. Wien, Math. Naturwiss. Kl. Abt. 2A, 145, 195-218 (1938)

[2] W. T. Koiter, General theorems for elastic-plastic solids, Progress in Solid Mechanics (I. N. Sneddon and R. Hill, editors), North-Holland, Amsterdam, 1960, pp. 165-221.

[3] D. A. Gokhfeld, Some problems of shakedown of plates and shells, Trudy VI Vsesoyuznoj Konf. Plastin i Obolochek, Izdat. Nauka, Moscow, 1966, pp. 284-291 (Russian)

[4] A. Sawczuk, Evaluation of upper bounds to shakedown loads for shells, J. Mech. Phys. Solids 17, 291-301 (1969)

[5] J. J. Moreau, Sur les lois de frottement, de viscosité et plasticité, C. R. Acad. Sci. Paris Sér. I. Math. 271, 608-611 (1970)

[6] O. Debordes, Duality: some results in asymptotical elastoplasticity, Convex Analysis and Its Application (A. Auslender, editor), Springer-Verlag, Berlin, 1977, pp. 100-114

[7] A. L. Goldenveizer, Theory of Elastic Thin Shells, Pergamon Press, New York, 1961

[8] P. P. Mosolov and V. P. Miasnikov, Asymptotic theory of rigid plastic shells, Prikl. Mat. Mekh. 41, 538-552 (1977)

[9] J. A. König, Shake-down of elastic-plastic structures, Elsevier, Amsterdam, 1987

[10] D. Weichert, Zum Problem geometrischer Nichtlinearitäten in der Plastizitätstheorie, Mitteilungen Institut Mechanik, vol. 53, Ruhr-Universität Bochum, 1987

[11] J. Groß-Weege, Zum Einspielverhalten von Flächentragwerken, Mitteilungen Institute für Mechanik, vol. 58, Ruhr-Universität Bochum, 1988

[12] W. Pietraszkiewicz, Introduction to the non-linear theory of shells, Mitteilungen Institut für Mechanik, vol. 10, Ruhr-Universität Bochum, 1977

[13] I. N. Vekua, Generalized Analytic Functions, Pergamon Press, New York, 1962 